\title{
Efficient Terahertz Detection with Perfectly-Absorbing Metasurface
}

\author{
Lucy Hale $^{1}$, Tom Siday ${ }^{1}$, Polina P. Vabishchevich ${ }^{2,3}$, Charles Thomas Harris ${ }^{2,3}$, Ting Shan Luk ${ }^{2,3}$, \\ John L. Reno ${ }^{2,3}$, Igal Brener ${ }^{2,3}$ and Oleg Mitrofanov ${ }^{1,2,3}$ \\ ${ }^{1}$ Electronic and Electrical Engineering, University College London, London, WC1E 7JE United Kingdom \\ ${ }^{2}$ Center for Integrated Nanotechnologies, Sandia National Laboratories, Albuquerque, New Mexico 87123, USA \\ ${ }^{3}$ Sandia National Laboratories, Albuquerque New Mexico 87185, USA
}

\begin{abstract}
We demonstrate a unique photoconductive design for terahertz (THz) detection based on a perfectly absorbing, alldielectric metasurface. Our design exploits Mie resonances in electrically connected cubic resonators fabricated in lowtemperature grown (LT) GaAs. Experimentally, the detector achieves very high contrast between ON/OFF conductivity states $\left(1^{7}\right)$ whilst also requiring extremely low optical power for optimal operation $(100 \mu \mathrm{W})$. We find that the Mie resonances dissipate sufficiently fast and maintain the detection bandwidth up to 3 THz.
\end{abstract}

\section{INTRODUCTION}

$\mathrm{P}$ HOTOCONDUCTIVE switches are instrumental to many $\mathrm{THz}$ imaging and spectroscopy systems. Their operation is based on the subpicosecond switching of electrical conductivity with incident optical light to generate or detect $\mathrm{THz}$ radiation [1]. For effective operation, it is vital that there is a high contrast between conductive and nonconductive states, and that the conversion rate of optical photons to charge carriers is high. Several approaches have been explored recently to modify optical properties of photoconductive switches and to improve their performance [2-4]. In particular, plasmonic nanostructures have been investigated by several groups; however it was found that plasmonic nanostructures may introduce significant Ohmic losses, which lead to reduction in efficiency and in the damage threshold [5].

In order to satisfy the photoconductive switch requirements without introducing ohmic losses, we nanostructure the LTGaAs photoconductive region to form an optically thin alldielectric photoconductive metasurface. It is capable of absorbing optical excitation at $800 \mathrm{~nm}$ without reflection or transmission [6]. This perfect absorption is achieved by exciting two degenerate, critically coupled modes of opposing symmetry with respect to the metasurface plane [7]. This is done by fabricating nanoscale cubic resonators that are tuned in size to support Mie resonances at $800 \mathrm{~nm}$. The first order magnetic dipole resonance, $M_{x}$ is directly excited by the incident beam (polarised in the y-direction). By introducing a bar structure, we break the cubic symmetry and allow excitation of the orthogonally oriented magnetic dipole, $M_{z}$. This mode is 'dark' in a symmetric resonator - it cannot be directly excited by a plane wave polarised in the resonator plane. The bar also electrically contacts the resonators, allowing charge carriers to be swept to the antennas for collection. In this way, optimal absorption is achieved without the use of a back reflector $[4,5]$, or plasmonic structures which increase ohmic losses [5] and may reduce the dark resistivity of the device. We emphasize that this is realised with a remarkably thin photoconductive layer of only $200 \mathrm{~nm}$, thereby increasing detection efficiency by reducing the transit distance of charge carriers to the antennae electrodes.

Here we investigate the properties of this structure as a $\mathrm{THz}$ photoconductive antenna detector, and evaluate effect of the perfectly absorbing metasurface on the $\mathrm{THz}$ detection properties.
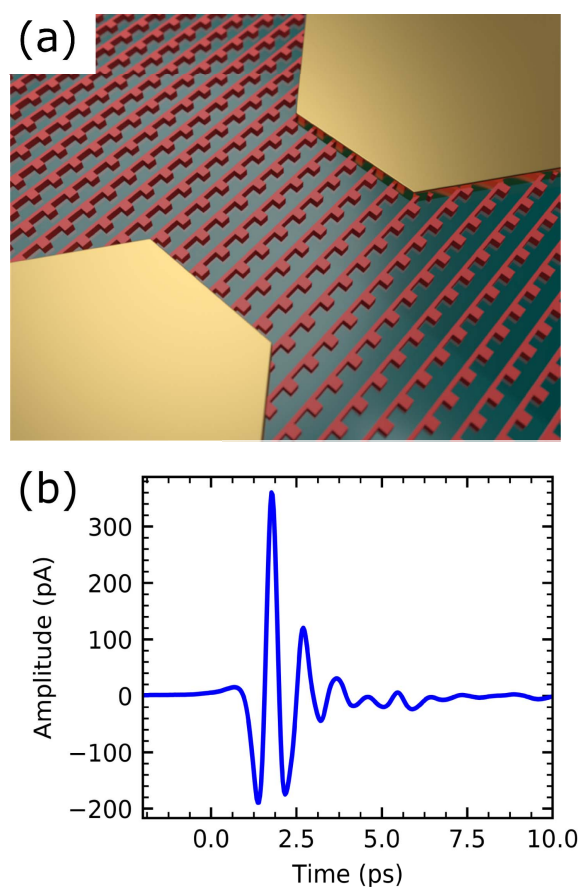

Fig 1. a) A conceptual illustration of the device showing the photoconductive metasurface (red) integrated into a photoconductive antenna detector. b) $\mathrm{THz}$ pulse measured from a $\mathrm{ZnTe}$ source using the device. [6]

\section{RESULTS}

Figure 1a shows a conceptual illustration of the device. The LT GaAs metasurface (red) is integrated between the electrodes for carrier collection. Experimentally we use the detector to measure waveforms of $\mathrm{THz}$ pulses from a $\mathrm{ZnTe}$ source (Fig.1b), and we achieve an optimal signal-to-noise (SNR) of $>10^{6}$. Moreover, this SNR is measured at an exceptionally low optical pump power of $100 \mu \mathrm{W}$ (Fig.2a). This early saturation onset is due to the extremely high absorption of the pump beam and high dark resistivity (50 $\mathrm{G} \Omega$ ) of the nanostructured active layer resulting in high ON/OFF contrast. Furthermore, a linear increase in RMS noise with optical power is observed, suggesting that the main source of noise is the laser power fluctuation rather than the detector itself.

We investigate potential limitations of using the perfect 
absorption technique for $\mathrm{THz}$ detection. The $M_{z}$ mode has a higher Q-factor than the $M_{x}$ mode, so when the modes are coupled the fields oscillate within the structure for longer before being absorbed. As a result, this could limit the detection bandwidth at high frequencies. We investigated this by looking at the frequency response of the detector for both $x$ and $y$-polarised light. For $y$-polarised light, both the $M_{x}$ and the $M_{z}$ modes are excited, whereas for $x$-polarised light it is the $M_{x}$ mode that is predominantly generated. The power spectral density (PSD) can be seen in Fig. 2b. At low frequencies, the $y$-polarisation PSD is higher than $x$-polarisation due to the increased optical absorption due to the excitation of the critically coupled modes. However, at higher frequencies, the detector performs worse in the $y$-polarisation due to the higher Q-factor and prolonged lifetime of the 'dark' $M_{z}$ mode. This is observed when using $\mathrm{THz}$ pulses from both InAs and $\mathrm{ZnTe}$ sources. Nevertheless, the effect of this on the detector performance is minimal and the bandwidth is still predominantly limited by the carrier recombination time within the photoconductive layer. The detector still maintains high sensitivity up to $3 \mathrm{THz}$.
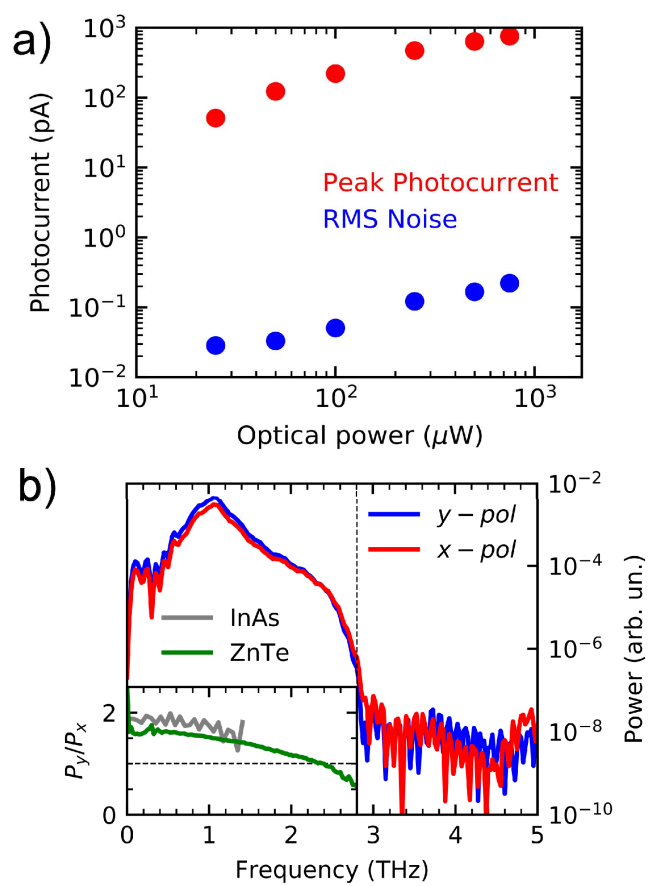

Fig. 2. a) Peak photocurrent (red) and RMS noise (blue) from the THz waveform measured for average optical pump power. b) Power spectral density (PSD) of the detected $\mathrm{THz}$ pulse waveforms for both $\mathrm{x}$ - and $\mathrm{y}$ polarizations of the optical gating pulses. The inset shows the PSD ratio for the two polarizations for frequencies up to $2.9 \mathrm{THz}$. The PSD ratio is measured using different $\mathrm{THz}$ pulses generated using InAs and $\mathrm{ZnTe}$ crystals (green, inset) to show that the ratio is independent of the $\mathrm{THz}$ pulse [6]

\section{SUMMARY}

In summary, we present an efficient photoconductive $\mathrm{THz}$ detector based on an all-dielectric metasurface. It enables perfect absorption whilst being optically thin and without the use of plasmonic structures. Experimentally, we achieve a maximum SNR of $>10^{6}$ and a detection bandwidth of $0.5-3$ $\mathrm{THz}$ using low optical powers of $100 \mu \mathrm{W}$.

\section{ACKNOWLEDGEMENTS}

This work was supported by the EPSRC (EP/L015277/1, EP/P021859/1, EP/L015455/1) and by the U.S. Department of Energy, Office of Basic Energy Sciences, Division of Materials Sciences and Engineering. Fabrication, optical, and in part $\mathrm{THz}$ experiments were performed at the Center for Integrated Nanotechnologies, an Office of Science User Facility operated for the U.S. Department of Energy (DOE) Office of Science. Sandia National Laboratories is a multimission laboratory managed and operated by National Technology and Engineering Solutions of Sandia, LLC., a wholly owned subsidiary of Honeywell International, Inc., for the U.S. Department of Energy's National Nuclear Security Administration under contract DE-NA0003525 .

\section{REFERENCES}

[1]. E. Castro-Camus, M. Alfaro, "Photoconductive Devices for Terahertz Pulsed Spectroscopy: A Review.” Photonics Res. 4, A36 (2016).

[2]. N.T. Yardimci, M. Jarrahi, "Nanostructure-Enhanced Photoconductive Terahertz Emission and Detection." Small 14 (44), 1802437 (2018).

[3]. S. Lepeshov, A. Gorodetsky, A. Krasnok, N. Toropov, T.A. Vartanyan, P. Belov, A. Alú, E.U. Rafailov, "Boosting Terahertz Photoconductive Antenna Performance with Optimised Plasmonic Nanostructures." Sci. Rep. 8(1), 6624 (2018).

[4]. O. Mitrofanov, T. Siday, R.J. Thompson, T.S. Luk, I. Brener, and J.L. Reno, "Efficient Photoconductive Terahertz Detector with All-Dielectric Optical Metasurface." APL Photonics 3, 51703-51703 (2018).

[5]. R.J. Thompson, T. Siday, S. Glass, T.S. Luk, J.L. Reno, I. Brener, and O. Mitrofanov, "Optically Thin Hybrid Cavity for Terahertz Photo-Conductive Detectors." Appl. Phys. Lett. 110 (4), 041105 (2017).

[6]. T. Siday, P.P. Vabischevich, L. Hale, C.T. Harris, T.S. Luk, J.L. Reno, I. Brener, I., and O. Mitrofanov, "Terahertz Detection with Perfectly-Absorbing Metasurface." Nano Lett. 19, 2888-2896 (2019).

DOI: 10.1021/acs.nanolett.8b05118.

[7]. X. Ming, X. Liu, L. Sun, and W. J. Padilla, "Degenerate Critical Coupling in All-Dielectric Metasurface Absorbers." Opt. Express 25 (20), 24658 (2017). 